\title{
Surveillance of SARS-CoV-2 in the environment and animal samples of the Huanan Seafood Market
}

\section{George Gao ( $\nabla$ gaof@im.ac.cn )}

Chinese Academy of Sciences https://orcid.org/0000-0002-3869-615X

\section{William Liu}

National Institute for Viral Disease Control and Prevention, Chinese Center for Disease Control and

Prevention https://orcid.org/0000-0003-3605-4070

\section{Peipei Liu}

MHC Key Laboratory of Biosafety, National Institute for Viral Disease Control and Prevention, China CDC

\section{Wenwen Lei}

National Institute for Viral Disease Control and Prevention, Chinese Center for Disease Control and Prevention

\section{Zhiyuan Jia}

NHC Key Laboratory of Biosafety, National Institute for Viral Disease Control and Prevention, Chinese Center for Disease Contol and Prevention

\section{Xiaozhou He}

NHC Key Laboratory of Biosafety, National Institute for Viral Disease Control and Prevention, Chinese Center for Disease Contol and Prevention

\section{Lin-Lin Liu}

Hubei Provincial Center for Disease Control and Prevention

\section{Weifeng Shi}

Key Laboratory of Etiology and Epidemiology of Emerging Infectious Disease in Universities of Shandong, Shandong First Medical University, and Shandong Academy of Medical Science

\section{Yun Tan}

Shanghai Institute of Hematology, State Key Laboratory of Medical Genomics, National Research Center for Translational Medicine, Ruijin Hospital Affiliated to Shanghai Jiao Tong School of Medicine

\section{Shumei Zou}

NHC Key Laboratory of Biosafety, National Institute for Viral Disease Control and Prevention, Chinese Center for Disease Contol and Prevention

\section{Xiang Zhao}

NHC Key Laboratory of Biosafety, National Institute for Viral Disease Control and Prevention, Chinese Center for Disease Contol and Prevention

\section{Gary Wong}

Public Health Agency of Canada

\section{Ji Wang}


NHC Key Laboratory of Biosafety, National Institute for Viral Disease Control and Prevention, Chinese Center for Disease Contol and Prevention

\section{Feng Wang}

NHC Key Laboratory of Biosafety, National Institute for Viral Disease Control and Prevention, Chinese Center for Disease Contol and Prevention

\section{Gang Wang}

NHC Key Laboratory of Biosafety, National Institute for Viral Disease Control and Prevention, Chinese Center for Disease Contol and Prevention

\section{Kun Qin}

NHC Key Laboratory of Biosafety, National Institute for Viral Disease Control and Prevention, Chinese Center for Disease Contol and Prevention

\section{Rongbao Gao}

NHC Key Laboratory of Biosafety, National Institute for Viral Disease Control and Prevention, Chinese Center for Disease Contol and Prevention

\section{Jie Zhang}

NHC Key Laboratory of Biosafety, National Institute for Viral Disease Control and Prevention, Chinese Center for Disease Contol and Prevention

\section{Min Li}

NHC Key Laboratory of Biosafety, National Institute for Viral Disease Control and Prevention, Chinese Center for Disease Contol and Prevention

\section{Wenling Xiao}

National Institute For Viral Disease Control and Prevention, Chinese Center For Disease Control and Prevention

\section{Yuanyuan Guo}

NHC Key Laboratory of Biosafety, National Institute for Viral Disease Control and Prevention, Chinese Center for Disease Contol and Prevention

\section{Ziqian Xu}

NHC Key Laboratory of Biosafety, National Institute for Viral Disease Control and Prevention, Chinese Center for Disease Contol and Prevention

\section{Yingze Zhao}

NHC Key Laboratory of Biosafety, National Institute for Viral Disease Control and Prevention, Chinese Center for Disease Contol and Prevention

\section{Jingdong Song}

National Institute For Viral Disease Control and Prevention, Chinese Center For Disease Control and Prevention https://orcid.org/0000-0001-8808-1981

\section{Jing Zhang}

NHC Key Laboratory of Biosafety, National Institute for Viral Disease Control and Prevention, Chinese Center for Disease Contol and Prevention

\section{Wei Zhen}


MHC Key Laboratory of Biosafety, National Institute for Viral Disease Control and Prevention, China CDC

\section{Wenting Zhou}

NHC Key Laboratory of Biosafety, National Institute for Viral Disease Control and Prevention, Chinese

Center for Disease Contol and Prevention

\section{Beiwei Ye}

NHC Key Laboratory of Biosafety, National Institute for Viral Disease Control and Prevention, Chinese Center for Disease Contol and Prevention

\section{Juan Song}

NHC Key Laboratory of Biosafety, National Institute for Viral Disease Control and Prevention, Chinese Center for Disease Contol and Prevention

\section{Mengjie Yang}

NHC Key Laboratory of Biosafety, National Institute for Viral Disease Control and Prevention, Chinese Center for Disease Contol and Prevention

\section{Weimin Zhou}

MHC Key Laboratory of Biosafety, National Institute for Viral Disease Control and Prevention, China CDC

\section{Yuhai Bi}

Institute of Microbiology, Chinese Academy of Sciences https://orcid.org/0000-0002-5595-363X

\section{Kun Cai}

Hubei Center for Disease Control and Prevention

\section{Dayan Wang}

NHC Key Laboratory of Biosafety, National Institute for Viral Disease Control and Prevention, Chinese Center for Disease Contol and Prevention

\section{Wenjie Tan}

China CDC https://orcid.org/0000-0002-5963-1136

\section{Jun Han}

MHC Key Laboratory of Biosafety, National Institute for Viral Disease Control and Prevention, China CDC

\section{Wenbo Xu}

MHC Key Laboratory of Biosafety, National Institute for Viral Disease Control and Prevention, China CDC

\section{Guizhen Wu}

National Institute for Viral Disease Control and Prevention, Chinese Center for Disease Control and Prevention

\section{Biological Sciences - Article}

Keywords: COVID-19, SARS-CoV-2, Huanan Seafood Market, zoonotic origin, cold chain, high-throughput sequencing, virus isolation

Posted Date: February 25th, 2022

DOI: https://doi.org/10.21203/rs.3.rs-1370392/v1 
License: (c) (i) This work is licensed under a Creative Commons Attribution 4.0 International License. Read Full License 
1 Surveillance of SARS-CoV-2 in the environment and animal samples of the

\section{Huanan Seafood Market}

\section{Running title:}

Prevalence of SARS-CoV-2 in Huanan Seafood Market

William J. Liu, ${ }^{1,7} \#$ Peipei Liu, ${ }^{1} \#$ Wenwen Lei, ${ }^{1} \#$ Zhiyuan Jia, ${ }^{1} \#$ Xiaozhou He, ${ }^{1} \#$ Linlin Liu ${ }^{2} \#$ Weifeng Shi, ${ }^{3} \#$ Y un Tan, ${ }^{4} \#$ Shumei Zou, ${ }^{1}$ Xiang Zhao, ${ }^{1}$ Gary Wong, ${ }^{5}$ Ji Wang, ${ }^{1}$ Feng Wang, ${ }^{1}$ Gang Wang, ${ }^{1}$ Kun Qin, ${ }^{1}$ Rongbao Gao, ${ }^{1}$ Jie Zhang, ${ }^{1}$ Min Li, ${ }^{1}$ Wenling Xiao, ${ }^{1,6}$ Yuanyuan Guo ${ }^{1,7}$, Ziqian Xu, ${ }^{1}$ Yingze Zhao, ${ }^{1}$ Jingdong Song, ${ }^{1}$ Jing Zhang, ${ }^{1}$ Wei Zhen, ${ }^{1}$ Wenting Zhou, ${ }_{1}$ Beiwei Ye, ${ }^{1}$ Juan Song, ${ }^{1}$ Mengjie Yang, ${ }^{1}$ Weimin Zhou, ${ }^{1}$ Yuhai Bi, ${ }^{8}$ Kun Cai, ${ }^{2}$ Dayan Wang, ${ }^{1}$ Wenjie Tan ${ }^{1}$, Jun Han ${ }^{1}$, Wenbo $\mathrm{Xu}^{1}$, George F. Gao, ${ }^{1,8}$ Guizhen $\mathrm{Wu}^{1}$

${ }^{1}$ NHC Key Laboratory of Biosafety, National Institute for Viral Disease Control and Prevention, Chinese Center for Disease Control and Prevention (China CDC), Beijing 102206, China;

${ }^{2}$ Hubei Provincial Center for Disease Control and Prevention, Wuhan, Hubei 430079, China;

${ }^{3}$ Key Laboratory of Etiology and Epidemiology of Emerging Infectious Diseases in Universities of Shandong, Shandong First Medical University, and Shandong Academy of Medical Sciences, Taian 271000, China;

${ }^{4}$ Shanghai Institute of Hematology, State Key Laboratory of Medical Genomics, National Research Center for Translational Medicine, Ruijin Hospital Affiliated to Shanghai Jiao Tong University (SJTU) School of Medicine, Shanghai 200020, China;

${ }^{5}$ CAS Key Laboratory of Molecular Virology \& Immunology, Institut Pasteur of Shanghai, Chinese Academy of Sciences (CAS), Shanghai 200031, China;

${ }^{6}$ School of Laboratory Medicine and Life Sciences, Wenzhou Medical University, Wenzhou 325035, China;

${ }^{7}$ School of Pharmaceutical Sciences, Nanjing Tech University, No. 30 South Puzhu Road, Nanjing 211816, China;

${ }^{8}$ CAS Key Laboratory of Pathogen Microbiology and Immunology, Institute of Microbiology, Chinese Academy of Sciences (CAS), Beijing 100101, China.

\# Contributed equally.

Correspondence:

George F. Gao: gaofu@chinacdc.cn

Guizhen Wu:wugz@ivdc.chinacdc.cn

William J. Liu: liujun@ivdc.chinacdc.cn 


\section{Abstract}

Emerging in December 2019, coronavirus disease 2019 (COVID-19) eventually became a pandemic and has posed a tremendous threat to global public health. However, the origins of SARS-CoV-2, the causative agent of COVID-19, remain to be determined. It has reported that a certain number of the early case clusters had a contact history with Huanan Seafood Market. Therefore, surveillance of SARS-CoV-2 within the market is of vital importance. Herein, we presented the SARS-CoV-2 detection results of 1380 samples collected from the environment and the animals within the market in early 2020. By SARS-CoV-2-specific RT-qPCR, 73 environmental samples tested positive for SARS-CoV-2 and three live viruses were successfully isolated. The viruses from the market shared nucleotide identity of $99.980 \%$ to $99.993 \%$ with the human isolate $\mathrm{HCoV} /$ Wuhan/IVDC-HB-01. In contrast, no virus was detected in the animal swabs covering 18 species of animals in the market. The SARS-COV-2 nucleic acids in the positive environmental samples showed significant correlation of abundance of Homo sapiens with SARS-CoV-2. In summary, this study provided convincing evidence of the prevalence of SARS-CoV-2 in the Huanan Seafood Market during the early stage of COVID-19 outbreak.

\section{Keywords:}

COVID-19, SARS-CoV-2, Huanan Seafood Market, zoonotic origin, cold chain, high-throughput sequencing, virus isolation 
Infections with novel human coronavirus 2019 (HCoV-19) (1, 2), named as severe acute respiratory syndrome coronavirus 2 (SARS-CoV-2) by International Committee on Taxonomy of Viruses (ICTV) (3), can result in coronavirus disease 2019 (COVID-19), characterized by various clinical outcomes from asymptomatic infections to severe pneumonia and even death (4-6). Globally, as of September 10th 2021, there have been 223,022,538 confirmed cases of COVID-19, including $4,602,882$ deaths. No sign hints the pandemic has declined. (covid19.who.int).

Early human cases with COVID-19 were first reported in late December 2019 from Wuhan, China with pneumonia of unknown etiology (PUE), and a majority of them were found to be linked to Huanan Seafood Market (HSM) in Wuhan (6, 7), where various animal meats and exotic seafoods were available for purchase. Therefore, HSM has been suspected to be the source of the COVID-19 pandemic (8). However, the possibility that some individuals outside the market had brought the virus into the market through human-to-human transmission or cold chain could not be ruled out, considering some of the early cases without epidemiological link to the market $(7,9)$. In addition, recent studies from different countries suggest that SARS-CoV-2 circulation preceded the initial detection of cases by weeks. Some of the suspected positive samples were detected even earlier than the first case in Wuhan, suggesting that potential circulation of the virus in other regions may have been missed(10-14).

Considering the findings that SARS-CoV-2 had high similarities with a few coronaviruses derived from bats in Asian countries including China, Japan, Cambodia, Thailand and Laos, scientists have proposed that bats might be the original source of SARS-CoV-2 (1, 15-20). However, it is yet a mystery whether another animal might act as an intermediate host to have facilitated the virus spillover between bats and humans (21). One of such important findings was the discovery of SARS-CoV-2 related coronaviruses from pangolins that harbored highly similar receptor binding domain with that of SARS-CoV-2 (22-24). Despite that pangolins might be involved 
in the ecology of coronaviruses, whether the pangolins are the intermediate hosts is still fairly controversial. A recent study documented the animal species in HSM between May 2017 and November 2019 and noted that no pangolins or bats were traded (25). Thus far, the origins of SARS-CoV-2 $(26,27)$ and the role of HSM in the origins and spread of SARS-CoV-2 remain poorly understood.

The HSM is located in the Jianghan District, a downtown of Wuhan, and is approximately $800 \mathrm{~m}$ far from Hankou Railway Station, a major railway travel hub. It occupies $>50,000 \mathrm{~m}^{2}$, with 678 stalls located close to each other in an extremely crowded condition (Fig. 1A). The market is separated into two zones, eastern and western zone, with seafood and animals mainly sold in the western zone and livestock meat in the eastern zone. Among the 678 stalls of the market, 10 domesticated wildlife animal-selling stalls $(1.5 \%)$ were identified, located in the south-western corner of western zone (8/10) and the north-western corner of eastern zone $(2 / 10)$, respectively (Fig. 1A). According to sales records, during late December 2019, animals or animal products were sold in these 10 animal stalls and animals included snakes, avian species (chickens, ducks, gooses, pheasants and doves), Sika deer, badgers, rabbits, bamboo rats, porcupines, hedgehogs, salamanders, giant salamanders, bay crocodiles and Siamese crocodiles, etc., among which snakes, salamanders and crocodiles were traded as live animals.

The market was closed in the morning of January $1^{\text {st }}, 2020$ shortly after the identification of the PUE. At the same time, in order to investigate the potential introduction of SARS-CoV-2 into the market, Chinese Center for Disease Control and Prevention (China CDC) dispatched an epidemiological team, together with experts from Hubei Provincial CDC and Wuhan Municipal CDC, to the HSM to collect environmental samples in the early morning of January $1^{\text {st }}, 2020$ (Fig. 1B). From January $1^{\text {st }}, 2020$ to March $2^{\text {nd }}, 2020$, a total of 923 environmental samples from different locations within and around this market and 457 animal samples including 
animal bodies, stray animals and their feces were collected, with some stray animals sampled until March 30 ${ }^{\text {th }}$ (Extended Data Table 1), which may reflect the profile of SARS-CoV-2 contamination in the market during the early phase of the outbreak. After the closure of the stalls in the market, the outside surface of the rolling shutter doors of the stalls and the corridors was disinfected (with $1 \%$ bleach mixed with water) throughout January and February 2020. The goods inside the stalls were completely cleared and disinfected until early March 2020.

Out of the 923 environmental samples collected in and around the market, 73 were found to be positive for SARS-CoV-2 with positive rate of $7.9 \%$ through the nucleic acid test (NAT), with Cycle threshold $(C t)$ values of real-time polymerase chain reaction (PCR) ranging from 23.9 to 41.7 (Table 1). Among the 828 samples inside the HSM, 64 samples $(7.7 \%)$ were positive. For the 14 samples from warehouses related to the HSM, five tested positive. Among the 51 sewerage wells in the surrounding areas, three tested positive (Table 1). Notably, one out of the 30 environmental samples from other markets in Wuhan tested positive.

For these 64 SARS-CoV-2 positive samples inside HSM, 87.5\% (56/64) were collected in the western zone of the market, in particular streets from no. 1 to 8 , with $71.4 \%(40 / 56)$ positive samples identified herein (Fig. 1A). Of the 110 samples collected from sewers or sewerage wells in the market, 24 samples were positive for SARS-CoV-2 nucleic acid. All the four sewerage wells in the market tested positive. This suggested that either contaminated sewage may have played a role in the cluster of cases in the market or that the infected people in the market contaminated the sewage.

The merchants' activities were assessed against the NAT results of the environmental samples. The sampling covered $19.8 \%(134 / 678)$ of the vendors in the market $(95 \%$ confidence interval (CI): 16.8-23.0\%). Of the positive samples, 44 were distributed 
among 21 vendors in the market, 19 of whom were located in the west zone and the remaining two located in the east area (Fig. 1A). Some vendors sold more than one product type, leading to differences in the denominators. While the results provide some indication of association of cases with different products, but no significant differences were observed between different vendors, including cold-chain products (18.4\%, 16/87, 95\% CI: 10.9-28.1\%), aquatic products $(17.8 \%, 13 / 73,95 \% \mathrm{CI}$ : 9.8-28.5\%), seafood products $(11 \%, 6 / 56: 95 \%$ CI: $4-21.9 \%)$, poultry $(22 \%, 8 / 37$ : 95\% CI: 9.8-38.2\%), livestock (14\%, 5/36: 95\% CI: 4.7-29.5\%), wildlife products (11\%, 1/9: 95\% CI: 0.3-48.2\%) and vegetables (25\%, 2/8: 95\% CI: 3.2-65\%) (Extended Data Fig. 1). These results suggested that SARS-CoV-2 might have been circulating in the market, especially the western zone, for a period of time in December 2019, leading to an extensive distribution of the virus within the market, which might have been facilitated by the crowded buyers and the contaminated environment.

The 457 animal samples mainly collected between January $1^{\text {st }}$ and March $2^{\text {nd }}, 2020$ included 188 individuals belonging to 18 species (with some stray animals sampled until March $30^{\text {th }}$ ) (Table 2). The sources of the samples include unsold goods kept in refrigerators and freezers in the stalls of HSM, and goods kept in warehouses and refrigerators related to the HSM. Samples from stray animals in the market were also collected, i.e. swab samples from 10 stray cats, 27 cat feces, one dog, one weasel, and 10 rats. All the 457 animal samples tested negative for SARS-CoV-2 nucleic acid, suggesting that the animal infections with SARS-CoV-2 might be rare in the market.

To determine whether there was live virus in the HSM, we inoculated 27 SARS-CoV-2 positive environmental samples collected on January $1^{\text {st }}, 2020$ into cell lines, including Vero E6 and Huh7.5 cells. Cytopathic effects (CPE) were observed 3 days post inoculation with sample F13 on Vero E6 cells. CPE was also observed 5 days post inoculation on Huh7.5 cells. We performed RT-qPCR on the supernatant of 
the inoculated Vero E6 and Huh7.5 cells. The supernatants were found to be SARS-CoV-2 positive, with $C t$ values of 15.11 (Vero E6) and 14.44 (Huh7.5) for the ORF1ab gene, and 13.98 (Vero E6) and 13.59 (Huh7.5) for the $\mathrm{N}$ gene, respectively. We further used transmission electron microscopy to observe the culture supernatant and ultra-thin section of Vero E6 cells 5 days post inoculation. The electron micrographs showed that virus particles were present in both the supernatant and the cells. Negative-stained virus particles were generally spherical, pleomorphic and 60-140 $\mathrm{nm}$ in diameter. Spike protrusions were observed around the particles in a crown (corona) shape. In the ultra-thin cultured cell sections, a group of virus particles could be seen outside the cells, and sheets of virus particles could also be observed inside the cells (Fig. 2). Totally, live viruses were isolated from samples F13, F54, and B5, which were the only three samples with $C t$ values $<30$ in the NAT. F54 and B5 were swab samples of the ground and F13 were swab samples of the wall. Notably, samples F13 and F54 were from the stalls with confirmed patients. All the results of successful virus isolation and the $C t$ values of the original samples revealed the existence of live SARS-CoV-2 with high titers in the environment of HSM.

We further performed high-throughput sequencing on the Miseq platform and successfully obtained seven complete SARS-CoV-2 genome sequences, including three sequences from three environmental samples (F13, F54, and A20), and four sequences from cell supernatants of F13, F54, and B5 (Fig. 3). The genome sequences of two environmental samples, F13 and F54, were found to be highly identical to the reference strain HCoV/Wuhan/IVDC-HB-01 (WH01, sequence identity of 99.993\%) and completely identical to the human stain Wuhan-Hu-1 (GenBank: NC_045512) (Fig. 3A). The genome sequence of the isolated virus from environmental sample F54 had two more synonymous mutations compared to HCoV/Wuhan/IVDC-HB-01, with sequence identity of $99.987 \%$ (Fig. 3A). Therefore, the SARS-CoV-2 sequences from environmental samples were highly similar to the clinical strains obtained during the early stage of the COVID-19 outbreak. 
210 Previously, SARS-CoV-2 has been proposed to be classified into two major

211 genotypes based on the two highly linked single nucleotide polymorphisms (SNPs): S 212 (8782T and 28144C) and L (8782C and 28144T), with S most likely to be the 213 ancestral lineage, because all of the SARS-CoV-2 related coronaviruses from bats and 214 pangolins possessed $8782 \mathrm{~T}$ and 28144C (28). Phylogenetic analysis revealed that 215 most of the environmental strains belong to the $\mathrm{L}$ lineage and they fall into the basal 216 positions of the L lineage (Fig. 3B). However, the environmental sample A20 fell 217 within the S lineage in the tree (Fig. 3B, Extended Data Table 2).

To explore the potential origins of the SARS-COV-2, we conducted RNA-seq analysis using 27 SARS-CoV-2 positive environmental samples collected on January $1^{\text {st }}, 2020$ from the HSM. We analyzed the correlation of SARS-CoV-2 and the abundance of other species. The abundance of Homo sapiens showed the correlation to SARS-CoV-2 (Figure 4), which highly suggests the SARS-CoV-2 might have derived from Homo sapiens in the HSM. No animals were concluded, implying that no animal host of SARS-CoV-2 can be deduced.

In summary, SARS-CoV-2 RNA was detected in stalls in the western zone of HSM, suggesting the prevalence of SARS-CoV-2 in the market. Thus, the market might have acted as an amplifier due to the high number of visitors every day, causing many initially identified infection clusters in the early stage of the outbreak as indicated in the Report of WHO-convened global study of origins of SARS-CoV-2 (10). In addition, live SARS-CoV-2 viruses also existed in the environmental samples. However, no SARS-CoV-2 was detected in the animal samples from the market. Definitely, more work involving international coordination is needed to investigate the real origins of SARS-CoV-2(10), especially considering the SARS-CoV-2 positive results of samples collected in 2019 in retrospective studies of different countries $(29,30)$. Surveillance of wild animals using a viromic approach should be 
enhanced to explore the natural and intermediate hosts for SARS-CoV-2 $(8,31)$, which would help to prevent future potential pandemics caused by animal-derived coronaviruses or alike.

\section{Acknowledgements}

We gratefully acknowledge the work and suggestions of the joint team of WHO-convened Global Study of Origins of SARS-CoV-2: China Part, including Chinese and international scientists and WHO experts. In addition, we also thank experts from Wuhan, Hubei Province and across China who contributed to the study.

\section{Author contributions}

249

The study was designed by W.J.L, G.F.G. and G-Z.W. Samples were collected by W.J.L., W.L, Z.J., X.H., J.W., F.W., G.W., K.Q., R.G., J.Z., M.L. and W.X. Experiment and data analysis were performed by W.J.L., P.L., Z.J., X.H., L.L., W.S., Y.T., S.Z., X.Z., J.W., F.W., G.W., Y.G., Z.X., Y.Z., J.S., Jing Z., W.Z., W-T.Z., B.Y., J.S., M.Y., W-M.Z., Y.B., K.C., D.W., W.T., J.H. and W-B.X. The manuscript was written by W.J.L., P.L., W.S., Y.T., G.W., G.F.G. and G-Z.W.

255

\section{Competing interest declaration}

257

No competing interest exists. 


\section{References}

261 1. W. Tan et al., A Novel Coronavirus Genome Identified in a Cluster of Pneumonia Cases Wuhan, China 2019-2020. China CDC Wkly 2, 61-62 (2020).

2632 2. S. Jiang et al., A distinct name is needed for the new coronavirus. Lancet 395, 949 (2020).

264 3. V. Coronaviridae Study Group of the International Committee on Taxonomy of, The species Severe acute respiratory syndrome-related coronavirus: classifying 2019-nCoV and naming it SARS-CoV-2. Nat Microbiol 5, 536-544 (2020).

4. W. J. Liu, G. Wu, Convincing the confidence to conquer COVID-19: From epidemiological intervention to laboratory investigation. Biosaf Health 2, 185-186 (2020).

5. N. Zhu et al., A Novel Coronavirus from Patients with Pneumonia in China, 2019. N Engl J Med 382, 727-733 (2020).

C. Wang, P. W. Horby, F. G. Hayden, G. F. Gao, A novel coronavirus outbreak of global health concern. Lancet 395, 470-473 (2020).

7. O. Li et al., Early Transmission Dynamics in Wuhan, China, of Novel Coronavirus-Infected Pneumonia. N Engl J Med 382, 1199-1207 (2020).

9. N. Chen et al., Epidemiological and clinical characteristics of 99 cases of 2019 novel coronavirus pneumonia in Wuhan, China: a descriptive study. Lancet 395, 507-513 (2020). Anonymous, $\mathrm{WHO}-$ convened global study of origins of SARS-CoV-2: China Part. https: / / www. who. int/publications/i/item/who-convened-global-study-of-origins-of -sars-cov-2-china-part (2021).

A. Eskild, L. Morkrid, S. B. Mortensen, T. M. Leegaard, Prevalence of antibodies against SARS-CoV-2 among pregnant women in Norway during the period December 2019 through December 2020. Epidemiol Infect 10.1017/S0950268822000073, 1-9 (2022).

G. Apolone et al., Unexpected detection of SARS-CoV-2 antibodies in the prepandemic period in Italy. Tumori 107, 446-451 (2021).

F. Carrat et al., Evidence of early circulation of SARS-CoV-2 in France: findings from the population-based "CONSTANCES" cohort. Eur J Epidemiol 36, 219-222 (2021).

J. C. Chandler et al., SARS-CoV-2 exposure in wild white-tailed deer lOdocoileus virginianus). Proc Natl Acad Sci US A 118 (2021).

$\mathrm{P}$. Zhou et al., A pneumonia outbreak associated with a new coronavirus of probable bat origin. Nature 579, 270-273 (2020).

S. Murakami et al., Detection and Characterization of Bat Sarbecovirus Phylogenetically Related to SARS-CoV-2, Japan. Emerg Infect Dis 26, 3025-3029 (2020).

S. Wacharapluesadee et al., Evidence for SARS-CoV-2 related coronaviruses circulating in bats and pangolins in Southeast Asia. Nat Commun 12, 972 (2021). H. Zhou et al., A Novel Bat Coronavirus Closely Related to SARS-CoV-2 Contains Natural Insertions at the S1/S2 Cleavage Site of the Spike Protein. Curr Bio/ 30, 2196-2203 e2193 (2020).

. H. Zhou et al., Identification of novel bat coronaviruses sheds light on the evolutionary origins of SARS-CoV-2 and related viruses. Cell 184, 4380-4391 e4314 (2021).

20. J. Li, S. Lai, G. F. Gao, W. Shi, The emergence, genomic diversity and global spread 
of SARS-CoV-2. Nature 600, 408-418 (2021).

21. R. Lu et al., Genomic characterisation and epidemiology of 2019 novel coronavirus: implications for virus origins and receptor binding. Lancet 395, 565-574 (2020).

22. T. T. Lam et al., Identifying SARS-CoV-2-related coronaviruses in Malayan pangolins. Nature 583, 282-285 (2020).

23. K. Xiao et al., Isolation of SARS-CoV-2-related coronavirus from Malayan pangolins. Nature 583, 286-289 (2020).

24. S. Niu et al., Molecular basis of cross-species ACE2 interactions with SARS-CoV-2-like

25. X. Xiao, C. Newman, C. D. Buesching, D. W. Macdonald, Z. M. Zhou, Animal sales

26. Q. Wang et al., Tracing the origins of SARS-CoV-2: lessons learned from the past. Cell

31. H. Li et al., Human-animal interactions and bat coronavirus spillover potential among rural

32. D. E. Wood, J. Lu, B. Langmead, Improved metagenomic analysis with Kraken 2. Genome Biol 20, 257 (2019). Res 31, 1139-1141 (2021).

27. Y. Tong et al., The origins of viruses: discovery takes time, international resources, and cooperation. Lancet 398, 1401-1402 (2021).

28. X. Tang et al., On the origin and continuing evolution of SARS-CoV-2. Nat/ Sci Rev 7 ,

29. G. Fongaro et al., The presence of SARS-CoV-2 RNA in human sewage in Santa Catarina,

30. G. Chavarria-Mir ó et al., Sentinel surveillance of SARS-CoV-2 in wastewater anticipates the occurrence of COVID-19 cases. medRxiv medrxiv. org/content/10.1101/2020.06.13.20129627v1 (2020). 


\section{Figure legends}

Figure 1. The distribution of the positive environmental samples in Huanan Seafood Market.

A. As the place of the early cluster of COVID-19 patients, the Huanan Seafood Market is separated into eastern and western zones with the Xinhua Rd. between them. To detect for the presence of SARS-CoV-2 RNA, reverse transcription, quantitative polymerase chain reaction (RT-qPCR) was performed. The locations of the positive samples were marked in the map of the market within orange, while the location of the samples that the live viruses were isolated from were labeled with red. The map also shows locations of stalls where domesticated wildlife products were sold. B. Timeline of environmental and animal samples collected within and around Huanan Seafood Market.

Figure 2. The SARS-CoV-2 virus isolation from environmental samples of Huanan Seafood Market.

The electron micrographs of the SARS-CoV-2 viruses isolated from the environmental samples in Huanan Seafood Market. To determine whether SARS-CoV-2 particles could be visualized from the cell supernatant and lysate, we used transmission electron microscopy to observe the culture supernatant and ultra-thin section cells based from both VeroE6 and Huh7.5 cells. The electron micrographs showed that virus particles were present in both the supernatant (A, B) and the cells (C, D). Negative-stained virus particles were generally spherical, pleomorphic and $60-140 \mathrm{~nm}$ in diameter. Spike protrusions were observed around the particles in a crown (corona) shape (A, B). In ultra-thin cultured cell sections, a group of virus particles can be seen outside the cell (C), and sheets of virus particles can also be observed inside the cells (D).

Figure 3. The genomic analysis of SARS-CoV-2 sequenced from Huanan Seafood Market. 
Figure 4. Clustering of environmental samples in Huanan Seafood Market.

363 A, Correlation of SARS-COV-2 with other species in SARS-COV-2 positive cases.

364 The abundance of SARS-COV-2 and other species was used for analysis. Both 365 Pearson's and Spearman's correlation values were plotted. B, Correlation of the 366 abundance of SARS-COV-2 and Homo sapiens.

367 
369 Table 1. Information of the positive environmental samples from Huanan Seafood

$370 \quad$ Market.

\begin{tabular}{|c|c|c|c|c|c|c|}
\hline No. & Sample type & Sampling date & PCR & $C t$ & PCR target & NGS \\
\hline E61 & Ground & $2020 / 1 / 1$ & + & 36.04 & ORF1ab/N & NA \\
\hline A101 & Surface of the door & $2020 / 1 / 1$ & + & 36.82 & ORF1ab/N & NA \\
\hline A14 & $\begin{array}{l}\text { Surface of packing bag for } \\
\text { hairtail }\end{array}$ & $2020 / 1 / 1$ & + & 36.42 & ORF1ab/N & NA \\
\hline A15 & Surface of the door & $2020 / 1 / 1$ & + & 35.51 & ORF1ab/N & NA \\
\hline A18 & Shoe covers and soles & $2020 / 1 / 1$ & + & 33.79 & ORF1ab/N & NA \\
\hline $\mathrm{A} 2$ & Ground & $2020 / 1 / 1$ & + & 35.07 & ORF1ab/N & + \\
\hline A20 & Gloves & $2020 / 1 / 1$ & + & 32.48 & ORF1ab/N & + \\
\hline A33 & Garbage truck & $2020 / 1 / 1$ & + & 34.46 & ORF1ab/N & NA \\
\hline A55 & Ground & $2020 / 1 / 1$ & + & 34.84 & ORF1ab/N & + \\
\hline A61 & Ground & $2020 / 1 / 1$ & + & 32.04 & ORF1ab/N & NA \\
\hline A63 & Ground & $2020 / 1 / 1$ & + & 34.43 & ORF1ab/N & + \\
\hline A 87 & Surface of the door & $2020 / 1 / 1$ & + & 36.94 & ORF1ab/N & NA \\
\hline A 88 & Ground & $2020 / 1 / 1$ & + & 36.69 & ORF1ab/N & NA \\
\hline A90 & Ground & $2020 / 1 / 1$ & + & 33.14 & ORF1ab/N & NA \\
\hline A96 & Ground & $2020 / 1 / 1$ & + & 33.97 & $\mathrm{ORF} 1 \mathrm{ab} / \mathrm{N}$ & NA \\
\hline B17 & Scale & $2020 / 1 / 1$ & + & 34.16 & $\mathrm{ORF} 1 \mathrm{ab} / \mathrm{N}$ & NA \\
\hline $\mathrm{B} 5^{*}$ & Ground & $2020 / 1 / 1$ & + & 29.32 & ORF1ab/N & + \\
\hline D32 & Surface of a cart & $2020 / 1 / 1$ & + & 33.72 & ORF1ab/N & NA \\
\hline E48 & $\begin{array}{l}\text { Blood on the ground in front of } \\
\text { the door }\end{array}$ & $2020 / 1 / 1$ & + & 35.93 & $\mathrm{ORF} 1 \mathrm{ab} / \mathrm{N}$ & + \\
\hline E7 & $\begin{array}{l}\text { Styrofoam desk in front of the } \\
\text { door }\end{array}$ & $2020 / 1 / 1$ & + & 36.44 & ORF1ab/N & + \\
\hline F100 & Ground & $2020 / 1 / 1$ & + & 34.72 & ORF1ab/N & + \\
\hline F13* & Surface of the wall & $2020 / 1 / 1$ & + & 23.85 & ORF1ab/N & + \\
\hline F33 & Roller shutter & $2020 / 1 / 1$ & + & 34.13 & ORF1ab/N & NA \\
\hline F46 & Ground & $2020 / 1 / 1$ & + & 31.8 & ORF1ab/N & + \\
\hline F54* & Ground & $2020 / 1 / 1$ & + & 25.8 & $\mathrm{ORF} 1 \mathrm{ab} / \mathrm{N}$ & + \\
\hline F98 & Ground & $2020 / 1 / 1$ & + & 34 & ORF1ab/N & + \\
\hline G93 & Sewage & $2020 / 1 / 1$ & + & 33.23 & ORF1ab/N & NA \\
\hline Q37 & Inner surface of the freezer & $2020 / 1 / 12$ & - & 1 & ORF1ab/N & + \\
\hline Q61 & Cart1 & $2020 / 1 / 12$ & - & 1 & ORF1ab/N & + \\
\hline Q64 & Cart2 & $2020 / 1 / 12$ & + & + & ORF1ab/N & + \\
\hline Q68 & Ground & $2020 / 1 / 12$ & + & + & ORF1ab/N & + \\
\hline Q69 & Feather removal machine & $2020 / 1 / 12$ & + & + & ORF1ab/N & + \\
\hline Q70 & Iron container in inner room & $2020 / 1 / 12$ & - & 1 & ORF1ab/N & + \\
\hline $1-27-33$ & Water drain & $2020 / 1 / 27$ & + & 36 & ORF $1 \mathrm{ab}$ & NA \\
\hline $1-27-37$ & Water drain & $2020 / 1 / 27$ & + & 35 & ORF1ab & NA \\
\hline $1-29-4$ & Water drain & $2020 / 1 / 29$ & + & 36 & ORF1ab & NA \\
\hline $1-29-8$ & Water drain & $2020 / 1 / 29$ & + & 37 & ORF1ab & NA \\
\hline $8-25-\mathrm{D}$ & Ground inside the stalls & $2020 / 2 / 3$ & + & 35.9 & ORF $1 \mathrm{ab}$ & + \\
\hline $8-25-\mathrm{M} 1$ & Ground inside the stalls & $2020 / 2 / 3$ & + & 36.5 & ORF $1 \mathrm{ab}$ & + \\
\hline WS-1-1 & West sewage well 1 & $2020 / 2 / 5$ & + & 36.00 & ORF $1 \mathrm{ab}$ & NA \\
\hline WS-1-2 & West sewage well 2 & $2020 / 2 / 5$ & + & 38.00 & ORF $1 \mathrm{ab}$ & NA \\
\hline WS-1-3 & West sewage well 3 & $2020 / 2 / 5$ & + & 34.01 & ORF $1 \mathrm{ab}$ & NA \\
\hline WS-1-4 & West sewage well 4 & $2020 / 2 / 5$ & + & 38.00 & ORF $1 \mathrm{ab}$ & NA \\
\hline WS-2-1 & West sewage well 1 & $2020 / 2 / 5$ & + & 37.30 & ORF1ab & NA \\
\hline WS-2-2 & West sewage well 2 & $2020 / 2 / 5$ & + & 37.65 & ORF $1 \mathrm{ab}$ & NA \\
\hline WS-2-3 & West sewage well 2 & $2020 / 2 / 5$ & + & 36.72 & ORF1ab & NA \\
\hline WS-3-2 & West sewage well 3 & $2020 / 2 / 5$ & + & 37.60 & ORF $1 \mathrm{ab}$ & NA \\
\hline WS-4-2 & West sewage well 4 & $2020 / 2 / 5$ & + & 37.10 & ORF $1 \mathrm{ab}$ & NA \\
\hline zong-1 & Water drain & $2020 / 2 / 9$ & + & 34.94 & ORF $1 \mathrm{ab}$ & NA \\
\hline$w-6-29-33$ & Water drain & $2020 / 2 / 15$ & + & 37.97 & ORF $1 \mathrm{ab}$ & NA \\
\hline w-zong-1 & Water drain & $2020 / 2 / 15$ & + & 36.54 & ORF $1 \mathrm{ab}$ & NA \\
\hline EWS-2\#-2 & East sewage well 2 & $2020 / 2 / 15$ & + & 35.32 & ORF $1 \mathrm{ab}$ & NA \\
\hline EWS-3\#-2 & West sewage well 3 & $2020 / 2 / 15$ & + & 36.05 & ORF $1 \mathrm{ab}$ & NA \\
\hline WWS-1\# & West sewage well 1 & $2020 / 2 / 15$ & + & 34.44 & ORF $1 \mathrm{ab}$ & NA \\
\hline WWS-1\#-2 & West sewage well 1 & $2020 / 2 / 15$ & + & 33.63 & ORF $1 \mathrm{ab}$ & NA \\
\hline
\end{tabular}




\begin{tabular}{lllllll}
\hline WWS-1\#-3 & West sewage well 1 & $2020 / 2 / 15$ & + & 33.58 & ORF1ab & NA \\
WWS-2\#-2 & West sewage well 2 & $2020 / 2 / 15$ & + & 37.44 & ORF1ab & NA \\
WWS-2\#-3 & West sewage well 2 & $2020 / 2 / 15$ & + & 36.88 & ORF1ab & NA \\
wws-1\#-0 & West sewage well 1 & $2020 / 2 / 15$ & + & 33.75 & ORF1ab & NA \\
W-8-25-D1 & Ground inside the stalls & $2020 / 2 / 15$ & + & 36.77 & ORF1ab & NA \\
W-8-25-D2 & Ground inside the stalls & $2020 / 2 / 15$ & + & 33.91 & ORF1ab & NA \\
W-8-25-L & Container & $2020 / 2 / 15$ & + & 34.58 & ORF1ab & NA \\
W-8-25-L2 & Container & $2020 / 2 / 15$ & + & 37.16 & ORF1ab & NA \\
C8 & Wall inside the stalls & $2020 / 2 / 20$ & + & $32.59 /-/ 37.34$ & RDRP/N/E & NA \\
SJ-D & Storehouse ground & $2020 / 2 / 22$ & + & $39.39 / 39.25 / 35.48$ & RDRP/N/E & NA \\
SJ-CS & Storehouse weight scale & $2020 / 2 / 22$ & + & $40.21 / 40.1 / 36.37$ & RDRP/N/E & NA \\
SJ-L3 & Storehouse wire fence & $2020 / 2 / 22$ & + & $41.77 / 41.62 / 37.61$ & RDRP/N/E & NA \\
RLC-4 & Storehouse bag surfaces & $2020 / 2 / 22$ & + & $41.71 / 41.53 / 37.31$ & RDRP/N/E & NA \\
RLC-3 & Storehouse bag surfaces & $2020 / 2 / 22$ & + & $36.18 / 36.05 / 32.36$ & RDRP/N/E & NA \\
\hline
\end{tabular}

371 Note: Four positive samples were not included in this table. One sample was collected from other market in Wuhan and 3 collected from sewerage wells in surrounding areas. 
374 Table 2. The animal samples collected in the Huanan Seafood Market.

\begin{tabular}{|c|c|c|c|c|}
\hline Species & Animal number & Sample number & $\begin{array}{l}\text { RT-PCR positive } \\
\text { number }\end{array}$ & Remarks \\
\hline Rabbit/Hares & 52 & 104 & 0 & \\
\hline Stray cat & 27 & $80^{\mathrm{a}}$ & 0 & Including faeces \\
\hline Snake & 40 & 80 & 0 & \\
\hline Hedgehog & 16 & 67 & 0 & \\
\hline Muntjac & 6 & 18 & 0 & \\
\hline Dog & 7 & 17 & 0 & $\begin{array}{l}\text { Including one } \\
\text { stray dog }\end{array}$ \\
\hline Badger & 6 & 16 & 0 & \\
\hline Bamboo rat & 6 & 15 & 0 & \\
\hline Rat & 10 & 12 & 0 & $\begin{array}{c}\text { Captured around } \\
\text { the market }\end{array}$ \\
\hline Pig & $\mathrm{NA}^{\mathrm{c}}$ & $6^{\mathrm{b}}$ & 0 & \\
\hline Chicken & 5 & 5 & 0 & \\
\hline $\begin{array}{l}\text { Chinese giant } \\
\text { salamander }\end{array}$ & 3 & 5 & 0 & \\
\hline Crocodile & 2 & 4 & 0 & \\
\hline Wild boar & 2 & 4 & 0 & \\
\hline $\begin{array}{l}\text { Soft-shelled } \\
\text { turtle }\end{array}$ & 2 & 3 & 0 & \\
\hline Weasel & 1 & 2 & 0 & $\begin{array}{l}\text { Captured around } \\
\text { the market }\end{array}$ \\
\hline Fish & 2 & 2 & 0 & \\
\hline Sheep & 1 & 1 & 0 & \\
\hline Others & $\mathrm{NA}^{\mathrm{c}}$ & 16 & 0 & \\
\hline Rabbit/Hares & 52 & 104 & 0 & \\
\hline Total & 188 & 457 & 0 & \\
\hline
\end{tabular}

$375{ }^{\text {a }}$ Six of the cats were from the Huanan Seafood Market.

376 b Other markets.

$377{ }^{\mathrm{c}}$ Not applicable. 


\section{Extended data methods}

\section{Sample collection}

Huanan Seafood Market (HSM) was closed on early morning of January 1st 2020 and at the same time, China CDC began collecting environmental and animal samples. Staff from China CDC entered the market about 30 times before the market's final clean-up on 2 March 2020, with some stray animals sampled outside the market until March $30^{\text {th }}$. Environmental samples in the HSM were collected to represent exhaustively as possible, from a wide diversity of surfaces, animals and products (Table 1 and 2) according to different sampling principles, as described in detail in the Joint Report of WHO-convened Global Study of Origins of SARS-CoV-2: China Part $(10)$.

The principles and ranges of in-market sampling covered: (1) environmental samples from stalls related to early cases; (2) environmental samples from doors and floors of all stalls in the blocks where the early cases were located; (3) environmental samples in the east wing of the market were collected according to blocks; (4) transport carts, trash cans and similar objects; (5) environmental samples from stalls that sold livestock, poultry, farmed wildlife (also called "domesticated wildlife" or "domesticated wildlife products" in this report); (6) samples of sewage and silt from drainage channels and sewerage wells; (7) stray cats, rats and other potential vector animals in the market; (8) animal products and other commodity samples kept in the cold storages and refrigerators in the market; (9) the market's ventilation and air-conditioning system; and (10) public toilets, public activity rooms and other places where people gathered in the market.

The investigators used full personal protective equipment during the sampling in the market. Commercial products of swabs and virus preservation solution were used for the sampling (Disposable Virus Sampling Tube, V5-S-25, Shen Zhen Zi Jian Biotechnology Co., Ltd., Shenzhen, China). For environmental samples, sampling swabs were applied to smear the floors, walls or surfaces of objects and then preserved them in virus preservation solution. 
For animal samples, depending on the type of animal and whether it was alive or frozen, pharyngeal, anal, body surface and body cavity swabs or tissue samples were collected for nucleic acid testing (NAT). Generally, for alive animal and frozen full bodies, three samples, including pharyngeal, anal, and body surface swabs were collected for each animal individuals. And for animal bodies after "bai tiao" disposing (remaining parts of poultry or livestock after removal of hair and viscera), the body cavity swabs were collected.

Sewage (silt) samples were collected by the use of virus sampling swabs to probe into the silt at the bottom of drainage channels in the market. Sewage and silt samples were preserved in virus preservation solution. For the sewage well, a container was used to take a silt-water mixture from a location near the bottom of the well, and an appropriate amount of sample was collected by using virus sampling swabs and then preserved in virus preservation solution.

\section{Nucleic acid extraction and SARS-CoV-2 real-time PCR assay}

A virus nucleic acid extraction kit (Xi'an Tianlong) was used to extract viral nucleic acid from samples using an automated nucleic acid extraction instrument according to the manufacturer's instructions. Real-time (RT) PCR was performed on extracted nucleic acid samples with a SARS-CoV-2 nucleic acid assay kit. The reagent brands include BioGerm (40/38, cycle number/cut-off value, the same as below), DAAN (45/40), and BGI (40/38).

\section{Virus isolations}

Samples positive for SARS-CoV-2 were cultured in Vero E6 and Huh7.5 cells. The cell lines were inoculated with positive samples and three blind passages were performed for each sample. The culture supernatant and cell pellet of each passage were harvested for RT PCR. The morphology of viral particles in the cell sections and the supernatant were observed by transmission electron microscope (TEM).

\section{Metagenomic sequencing}

Metagenomic sequencing was conducted at Wuhan BGI. Nucleic acid was extracted using Qiagen's viral RNA microextraction kit and human nucleic acid was removed 
436 using an enrichment kit to improve the sensitivity of viral RNA detection. Extracted 437 RNA was reverse transcribed into cDNA and segmented into 150-200 bp by enzyme 438 digestion. After repair, fitting, purification, PCR amplification and purification, 439 sample concentration was assayed and SE50+10 sequencing performed by 440 DNBSEQ-T7, and an average output of more than 200 million reads was obtained. 441 Sequencing data were compared with those in a SARS-CoV-2 database to determine 442 whether the samples contained coronavirus sequences.

443 Identification of the species abundances

444 The species abundances were obtained from the RNA-seq datasets of the 445 environmental samples. The raw sequenced reads were mapped to the NCBI 446 Nucleotide database using the kraken2 software (confidence 0.05) (32). The matrix of 447 species was obtained by using the pavian algorithm (33). The correlation value 448 between SARS-COV-2 and other species were examined by the Pearson's and 449 Spearman's correlation coefficient method. 
Extended Data Figure S1. Positive environmental samples associated with different products in the Huanan Seafood Market.

455 Dots represent the percentage of positive environmental samples associated with each product. Bars represent $95 \%$ confidence intervals for the binomials in the text above.

Note that the CI for some products (e.g. vegetables, farmed wildlife) have broad error bars that are likely due to the low number of vendors for these categories in the market. Nine of the 10 vendors selling farmed wildlife have been sampled.

Extended Data Table 1. Overview of environmental sample sampling and testing in the Huanan Seafood Market.

\begin{tabular}{|c|c|c|c|}
\hline & $\begin{array}{l}\text { Number of } \\
\text { samples }\end{array}$ & $\begin{array}{l}\text { Number of positive } \\
\text { samples by RT-PCR }\end{array}$ & $\begin{array}{l}\text { Number of } \\
\text { isolated viruses }\end{array}$ \\
\hline Huanan Seafood Market & 718 & 40 & 3 \\
\hline $\begin{array}{l}\text { Warehouses related to the } \\
\text { Huanan Seafood Market }\end{array}$ & 14 & 5 & \\
\hline Other markets in Wuhan* & 30 & 1 & \\
\hline $\begin{array}{l}\text { Drainage system in the Huanan } \\
\text { Seafood Market }\end{array}$ & 110 & 24 & \\
\hline $\begin{array}{l}\text { Sewerage wells in surrounding } \\
\text { areas }\end{array}$ & 51 & 3 & \\
\hline Total & 923 & 73 & 3 \\
\hline
\end{tabular}

*The other markets were Dongxihu market and Huanggang Center market.

Extended Data Table 2. Summary of number of reads mapped to positions 8782 and 28144 in different samples.

\begin{tabular}{|c|c|c|c|c|c|c|c|c|}
\hline \multirow{2}{*}{ Position } & \multicolumn{4}{|c|}{8782} & \multicolumn{4}{|c|}{28144} \\
\hline & $\mathrm{A}$ & $\mathrm{T}$ & $\mathrm{C}$ & $\mathrm{G}$ & A & $\mathrm{T}$ & $\mathrm{C}$ & G \\
\hline A101 & 0 & 0 & 0 & 0 & 0 & 0 & 0 & 0 \\
\hline A14 & 0 & 0 & 0 & 0 & 0 & 0 & 0 & 0 \\
\hline A15 & 0 & 0 & 0 & 0 & 0 & 0 & 0 & 0 \\
\hline A18 & 0 & 0 & 0 & 0 & 0 & 0 & 0 & 0 \\
\hline $\mathrm{A} 20$ & 7 & 359 & 1 & 0 & 1 & 5 & 1596 & 5 \\
\hline A2 & 0 & 0 & 0 & 0 & 0 & 0 & 0 & 0 \\
\hline A33 & 0 & 0 & 2 & 0 & 0 & 0 & 1 & 0 \\
\hline A55 & 0 & 0 & 0 & 0 & 0 & 0 & 0 & 0 \\
\hline A61 & 0 & 0 & 2 & 0 & 0 & 0 & 1 & 0 \\
\hline A63 & 0 & 0 & 0 & 0 & 0 & 0 & 0 & 0 \\
\hline A87 & 0 & 0 & 0 & 0 & 0 & 1 & 0 & 0 \\
\hline
\end{tabular}




\begin{tabular}{lrrrrrrrr}
\hline A88 & 0 & 0 & 0 & 0 & 0 & 2 & 0 & 0 \\
A90 & 0 & 0 & 0 & 0 & 0 & 0 & 0 & 0 \\
A96 & 0 & 0 & 0 & 0 & 0 & 0 & 0 & 0 \\
B17 & 0 & 0 & 0 & 0 & 0 & 0 & 0 & 0 \\
B5 & 0 & 0 & 86 & 0 & 0 & 85 & 0 & 0 \\
D32 & 0 & 0 & 0 & 0 & 0 & 0 & 0 & 0 \\
E48 & 0 & 0 & 0 & 0 & 0 & 0 & 0 & 0 \\
E61 & 0 & 0 & 0 & 0 & 0 & 0 & 0 & 0 \\
E7 & 0 & 0 & 0 & 0 & 0 & 0 & 0 & 0 \\
F100 & 0 & 0 & 0 & 0 & 0 & 0 & 0 & 0 \\
F13 & 5 & 10 & 1874 & 3 & 0 & 1669 & 0 & 0 \\
F33 & 0 & 0 & 0 & 0 & 0 & 0 & 0 & 0 \\
F46 & 0 & 0 & 1 & 0 & 0 & 0 & 0 & 0 \\
F54 & 1 & 1 & 106 & 0 & 1 & 39 & 0 & 0 \\
F98 & 0 & 0 & 20 & 0 & 0 & 3 & 0 & 0 \\
G93 & 0 & 0 & 0 & 0 & 0 & 0 & 0 & 0 \\
\hline
\end{tabular}




\section{Figures}

A

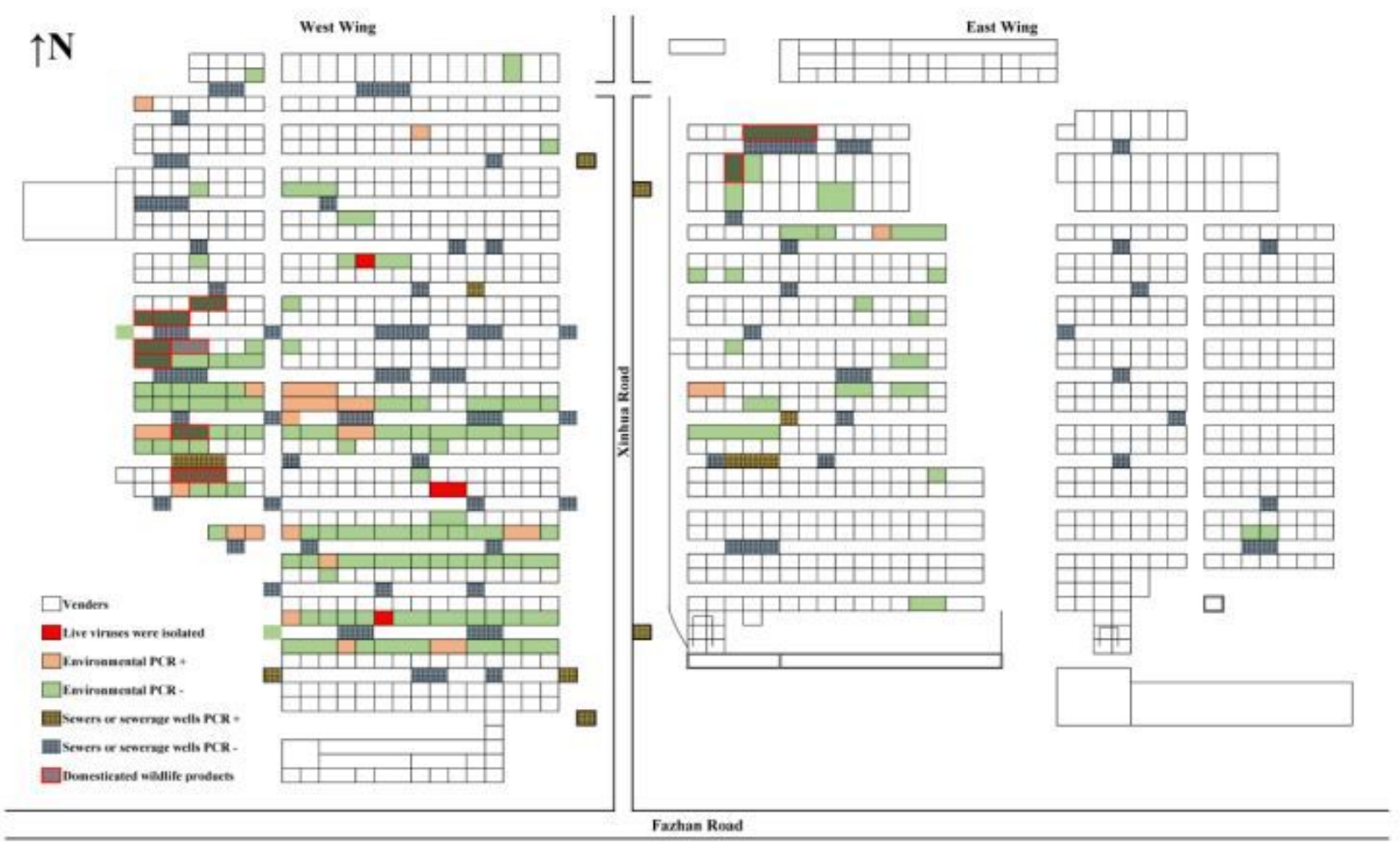

B

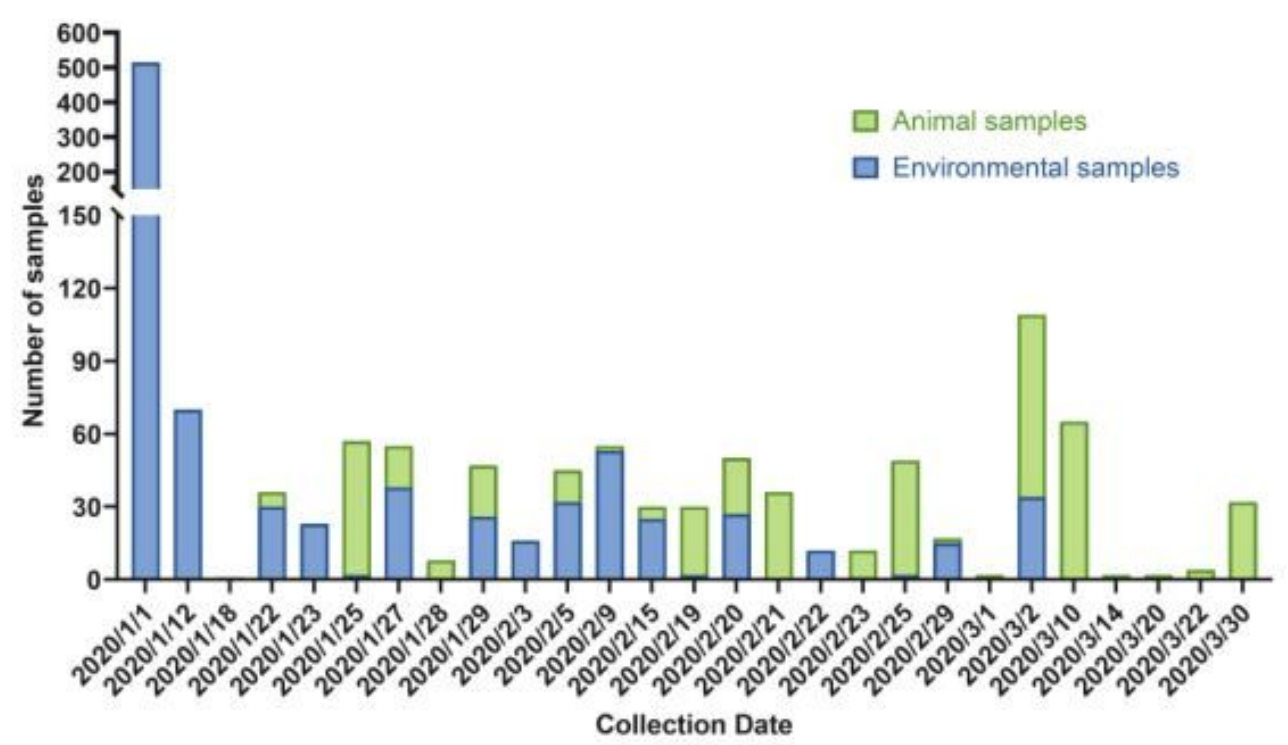

Figure 1

The distribution of the positive environmental samples in Huanan Seafood Market. A. As the place of the early cluster of COVID-19 patients, the Huanan Seafood Market is separated into eastern and western zones with the Xinhua Rd. between them. To detect for the presence of SARS-CoV-2 RNA, reverse 
transcription, quantitative polymerase chain reaction (RT-qPCR) was performed. The locations of the positive samples were marked in the map of the market within orange, while the locationof the samples that the live viruses were isolated from were labeled with red. The map also shows locations of stalls where domesticated wildlife products were sold. B. Timeline of environmental and animal samples collected within and around Huanan Seafood Market
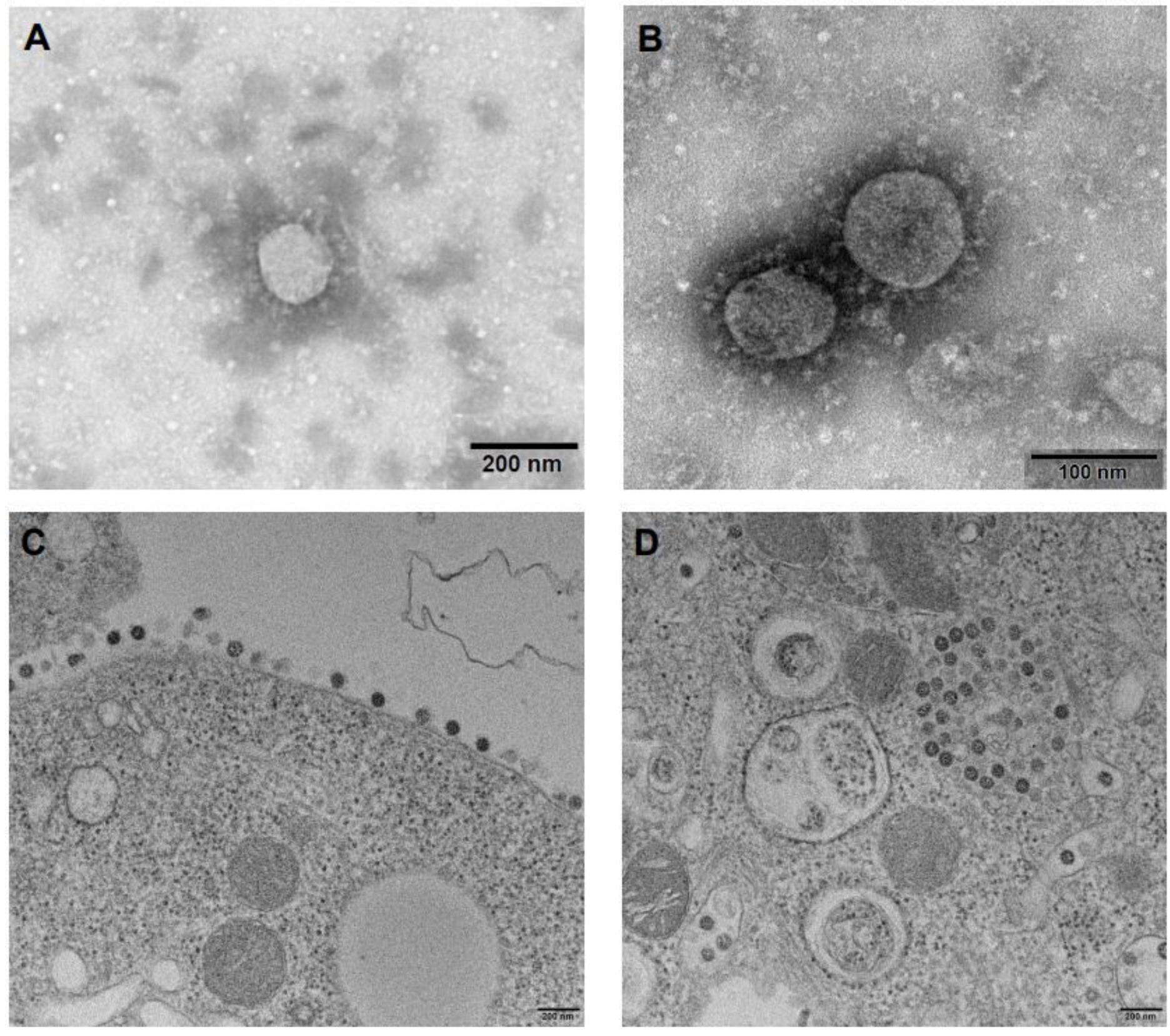

\section{Figure 2}

The SARS-CoV-2 virus isolation from environmental samples of Huanan Seafood Market. The electron micrographs of the SARS-CoV-2 viruses isolated fromthe environmental samples in Huanan Seafood Market. To determine whether SARS-CoV-2 particles could be visualized from the cell supernatant and 
lysate, we used transmission electron microscopy to observe the culture supernatant and ultra-thin section cells based from both VeroE6 and Huh7.5 cells. The electron micrographs showed that virus particles were present in both the supernatant (A, B) and the cells (C, D). Negative-stained virus particles were generally spherical, pleomorphic and $60-140 \mathrm{~nm}$ in diameter. Spike protrusions were observed around the particles in a crown (corona) shape (A, B). In ultra-thin cultured cell sections, a group of virus particles can be seen outside the cell (C), and sheets of virus particles canalso be observed inside the cells (D).

A
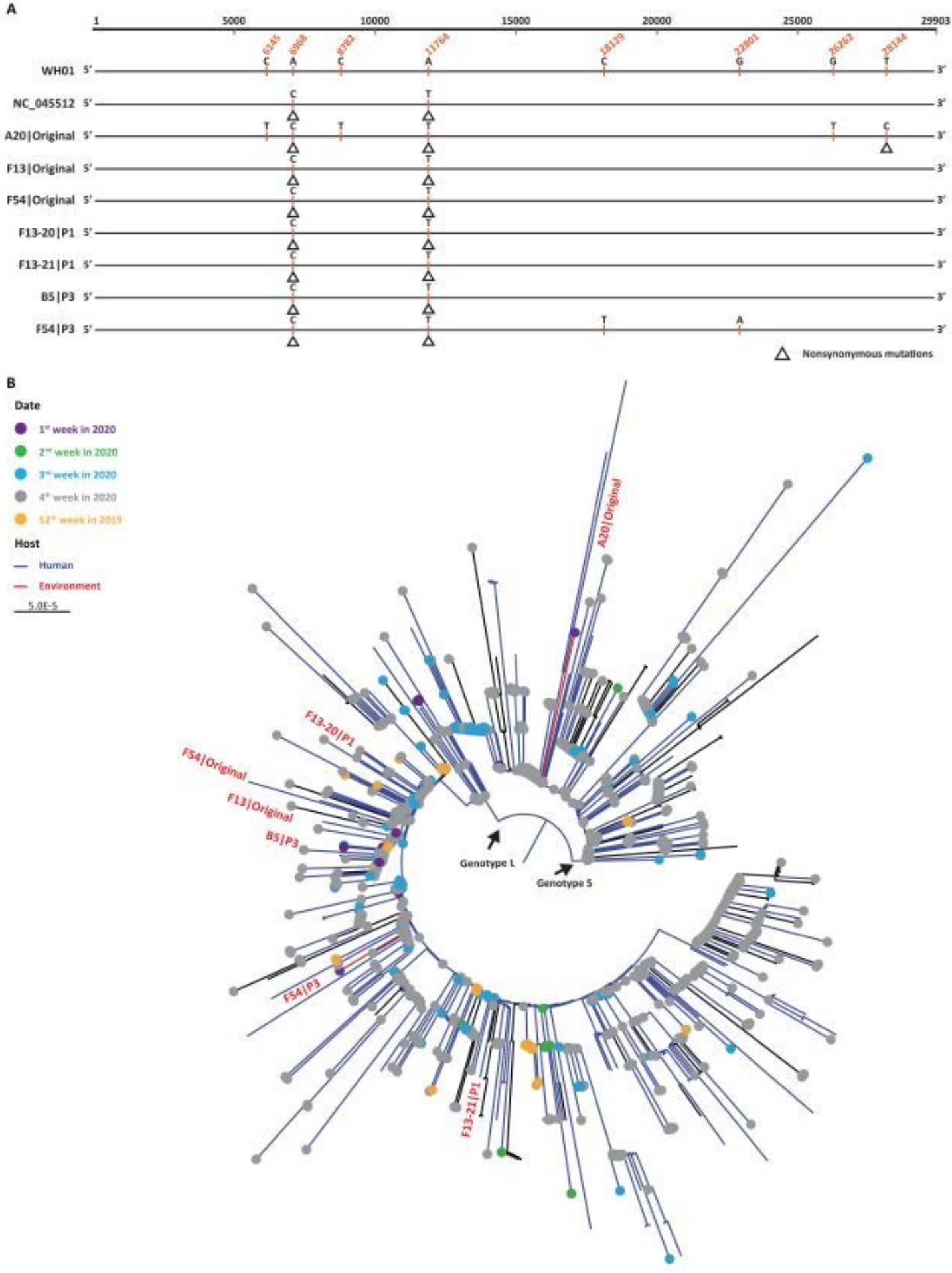
Figure 3

The genomic analysis of SARS-CoV-2 sequenced fromHuanan Seafood Market.

A

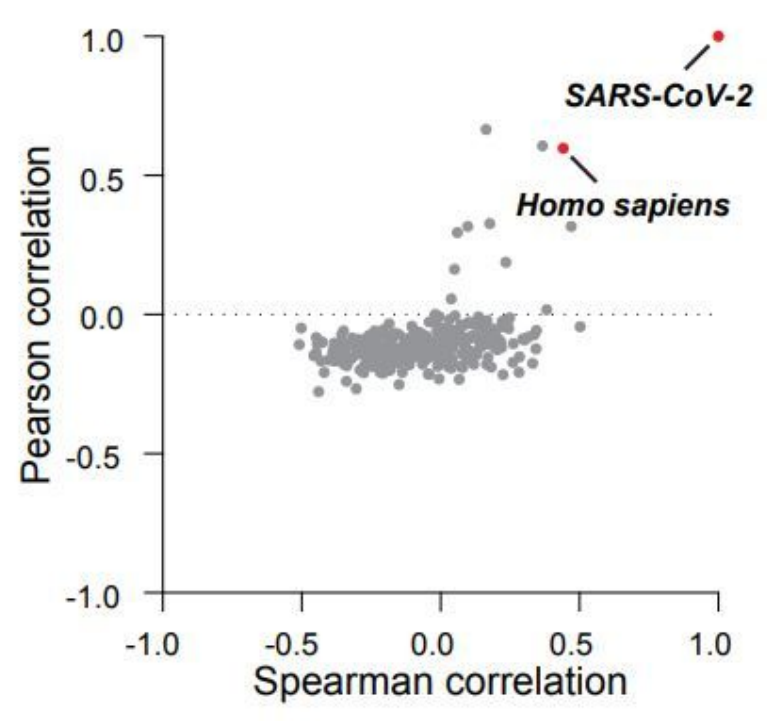

B

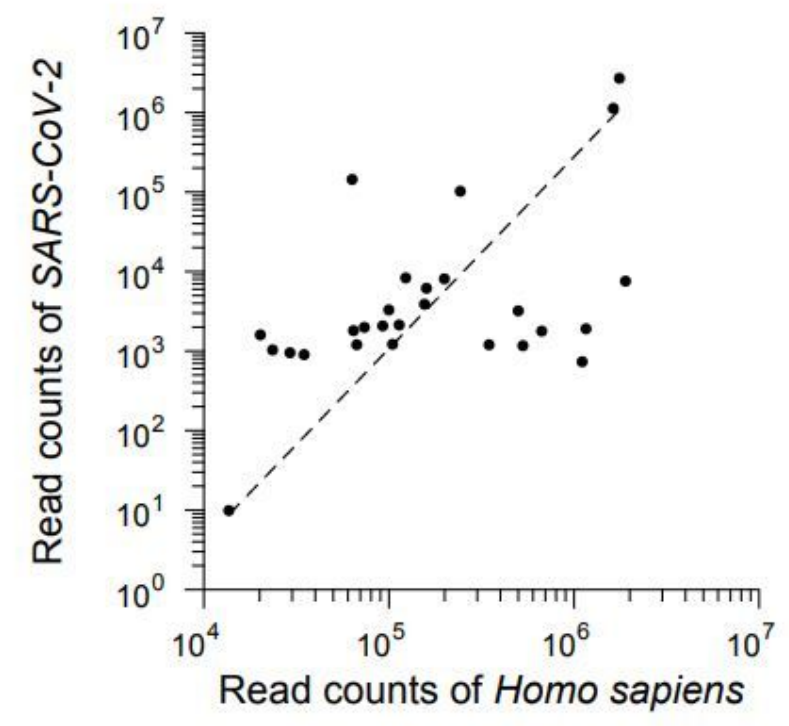

\section{Figure 4}

Clustering of environmental samples in Huanan Seafood Market. A, Correlation of SARS-COV-2 with other species in SARS-COV-2 positive cases. The abundance of SARS-COV-2 and other species was used for analysis. Both Pearson's and Spearman's correlation values were plotted. B, Correlation of the abundance of SARS-COV-2 and Homo sapiens. 